How I do it

coloproctology 2021 · 43:114-118

https://doi.org/10.1007/s00053-021-00519-3

Angenommen: 14. Januar 2021

Online publiziert: 12. Februar 2021

(c) Springer Medizin Verlag $\mathrm{GmbH}$, ein Teil von Springer Nature 2021

\section{Der Pilonidalsinus oder Sinus pilonidalis (pilus $=$ Haar, nidus $=$ Nest) ist eine akut abszedierende oder chronisch sezernierende Entzündung im subkutanen Fettgewebe im Bereich der Steißbeinregion. In vielen Fällen ist der Sinus pilonidalis asymptomatisch und bedarf keiner Therapie. Der Begriff wurde nach der Erstbeschreibung 1833 durch Mayo und Andersons Publikation „Hair extracted from an ulcer“ (1847), später dann 1880 von Hodges geprägt.}

Die Häufigkeit des Sinus pilonidalis wird mit 26/100.000 Einwohner angegeben. Das Krankheitsbild tritt meist im 2. bis 3. Lebensjahrzehnt auf, häufig bei Männern mit ausgeprägter Behaarung in der Glutealregion vor dem 40. Lebensjahr.

Der Pilonidalsinus wird heute als eine vornehmlich in der Pubertät erworbene Erkrankung bei möglicherweise genetischer Disposition angesehen. Seiner Entstehung scheint ein multifaktorielles Geschehen, ausgelöst durch folgenden $\mathrm{Me}-$ chanismus zugrunde zu liegen: das Aneinanderreiben der Nates in der Rima ani. Die Nates drehen abgebrochene Haare mit ihren wurzelnahen Enden in die Haut hinein. Dadurch entstehen sog. Pori oder Pits, d. h. Vertiefungen, die Haare enthalten können. Da die Hornschuppen der Haare als Widerhaken fungieren, dringt das Haar immer tiefer bis in das subkutane Fettgewebe ein. Dort entwickelt sich ein Fremdkörpergranulom, das nicht spontan heilt (asymptomatische Form), sich aber infizieren kann (abszedierende und chronische Form). Starke Behaarung sowie und übermäßige Schweißsekretion scheinen die Entstehung des Pilonidalsinus zu begünstigen.

Franc H. Hetzer

Viszeralchirurgie/Koloproktologie, Praxis Bellaria/Klinik im Park, Zürich, Schweiz

\title{
Modifizierter Limberg-Lappen beim Sinus pilonidalis
}

Die Beschwerden sind vom Erscheinungsbild abhängig: Die asymptomatische Form ist durch eine oder mehrere reizlose Pori (engl.: „pits“) in der Rima ani gekennzeichnet und wird nur zufällig diagnostiziert. Sie wird definiert durch den Nachweis von Pori ohne aktuelle oder vorhergehende relevante Beschwerden (Schmerzen, Sekretion, Blutung). Die akut abszedierende Form imponiert mit Schwellung und Schmerzen meist paramedian der Rima ani. Nach Spontanperforation oder chirurgischer Spaltung entleert sich Eiter.

Im chronischen Stadium leiden die Patienten unter permanenten oder intermittierenden serös-eitrigen Sekretionen aus dem Porus selbst (Pit, Primäröffnung) bzw. aus den lateralen Sekundäröffnungen.

\section{》) Ein blander Sinus pilonidalis bedarf keiner Therapie}

Ein blander Sinus pilonidalis bedarf keiner Therapie. Bei der akut abszedierenden Form gibt es Hinweise darauf, dass zunächst eine Abszessabdeckelung, gefolgt von einer sekundären Versorgung des Sinus pilonidalis im entzündungsfreien Intervall durch eine der weiter unten beschriebenen Behandlungsmethoden vorteilhaft ist. Beim chronischen Sinus pilonidalis stellt die Exzision derzeit die Standardbehandlung dar. Die offene Wundbehandlung mit sekundärer Wundheilung ist hinsichtlich der Komplikationsrate ein sicheres Verfahren, das aber mit einer langen Wundheilungsdauer und einer nicht zu vernachlässigender Rezidivrate einhergeht. Die minimal-invasiven Techniken - u. a. das sog. Pit-Picking - stellen ebenfalls eine
Behandlungsoption für die chronische Erscheinungsform dar, wobei mit einer höheren Rezidivrate im Vergleich zu den Exzisionstechniken gerechnet werden muss. Sie können aber als erste Methode bei unkompliziertem Pilonidalsinus eingesetzt werden. Die Anwendung des Mittellinienverschlusses zur primären Wunddeckung ist mit deutlich schlechteren Ergebnissen verbunden und somit obsolet. Wenn minimal-invasive Methoden nicht zum Einsatz kommen, sollten die plastischen (asymmetrischen) Techniken und Verschiebelappen verwendet werden. Limberg- und Karydakis-Lappen sind die derzeit am häufigsten angewendeten Verfahren und hinsichtlich der Ergebnisse gleichwertig.

Die Limberg-Plastik ist die am häufigsten beschriebene und am besten analysierte plastische Operationsmethode des Sinus pilonidalis. Zugleich stellt sie die wohl am häufigsten in Deutschland angewendete plastische Behandlungsmethode des Sinus pilonidalis dar. Die Limberg-Plastik wurde 1948 von dem sowjetischen Mund-Kiefer-Gesichts-Chirurgen Alexander Limberg beschrieben. Einer rautenförmigen Exzision des Sinusgewebes folgt die Mobilisation eines ebenfalls rautenförmigen subkutanen Lappens, mit dem der Defekt gedeckt wird. Die Anwendung der LimbergPlastik bei Patienten mit einem Sinus pilonidalis wurde zum ersten Mal von Gwynn beschrieben.

Durch die Limberg-Plastik wird die Rima ani abgeflacht und die Wunde lateralisiert. Diese beiden Umstände verhindern wahrscheinlich das Rezidiv. Die untere Ecke der Raute ist ein Schwachpunkt des Verfahrens, wenn er, wie ursprünglich beschrieben, in der Rima ani zu liegen kommt. Es kommt dann nicht 


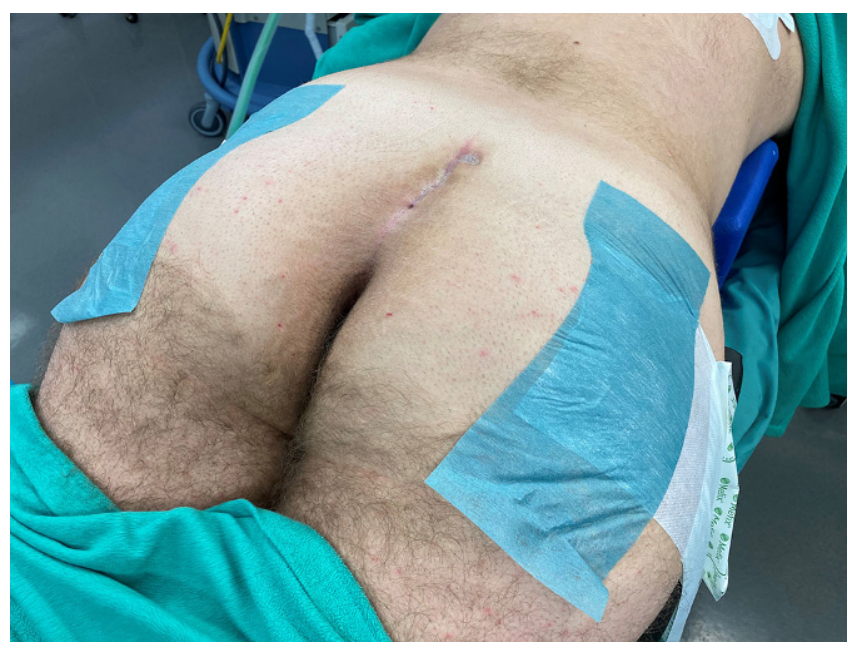

Abb. 1 \& Lagerung des Patienten auf dem Operationstisch

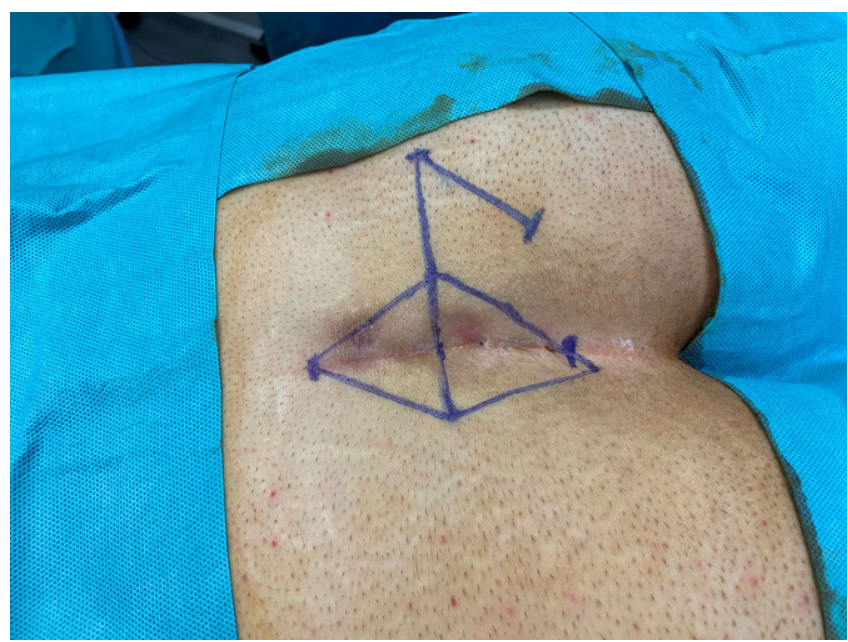

Abb. 3 A Einzeichnen der Exzisonsraute und des Verschiebelappens

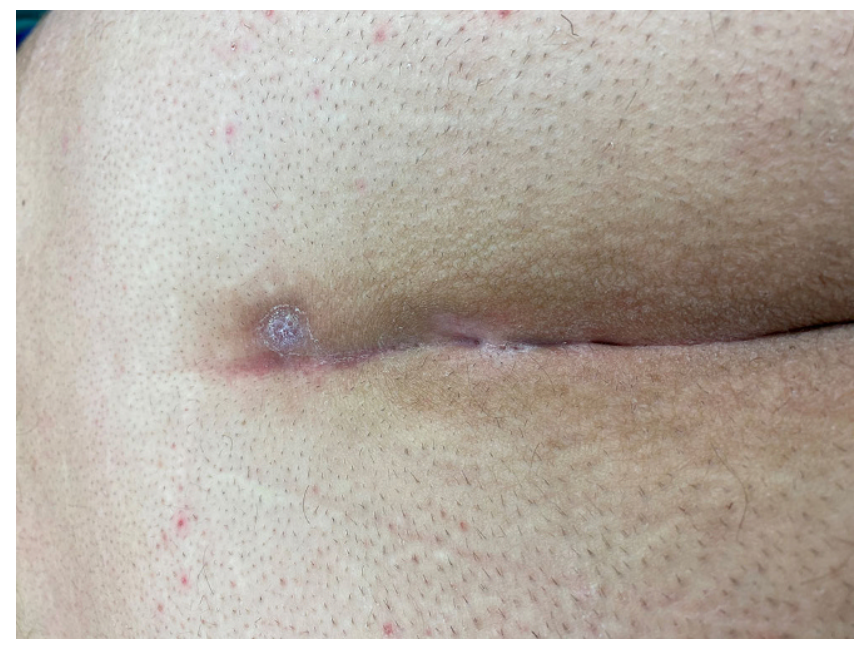

Abb. $2 \Delta$ Rima ani mit mehreren reizlosen Sinus pilonidales distal und einer Narbe proximal paramedian rechts nach Abdeckelung eines Abszesses

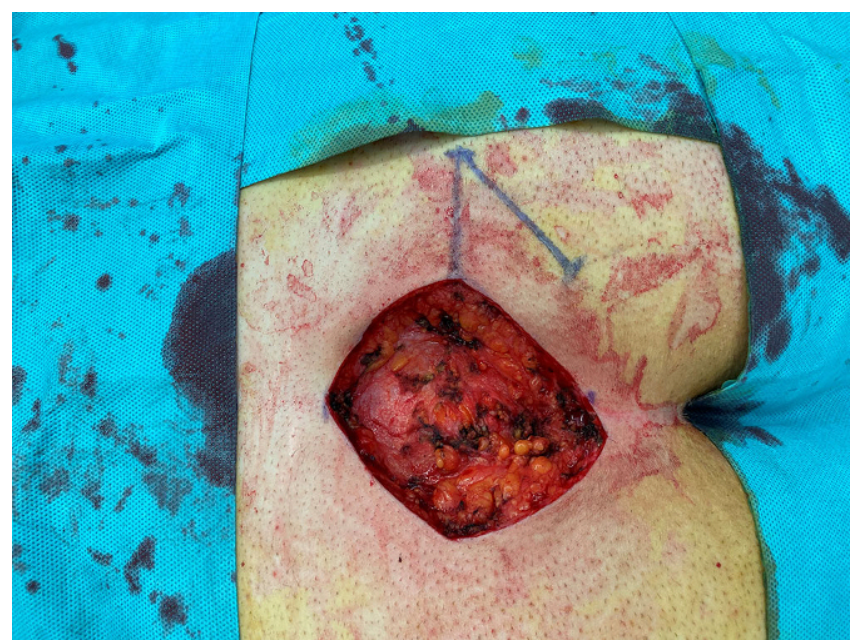

Abb. $4 \Delta$ Komplette Exzision des Sinus pilonidalis selten zu Wundheilungsstörungen oder Rezidiven. Von zahlreichen Autoren wurde deshalb die Methode modifiziert und fortan als modifizierte Limberg-Plastik bezeichnet: Der Unterpol der zu exzidierenden Raute wird dabei etwas aus der Rima ani verlagert.

\section{》) Die Rezidivrate nach der modifizierten Limberg-Plastik liegt unter $5 \%$}

In den über 50 veröffentlichten Arbeiten zu der Limberg-Plastik werden durchweg niedrige Rezidivraten von $0-6 \%$ demonstriert. Die Rezidivraten nach der modifizierten Limberg-Plastik scheinen noch niedriger zu sein (0-3\%).
Die Wunddehiszenzrate nach der Limberg-Plastik liegt bei 0-45\%, wobei häufiger über eine Inzidenz zwischen 5 und $15 \%$ berichtet wird [1].

\section{Perioperatives Management}

Die Patienten werden ohne Darmvorbereitung am Operationstag hospitalisiert. Der Eingriff wird in Bauchlage entweder in Allgemeinnarkose oder Spinalanästhesie durchgeführt und dauert in der Regel $60 \mathrm{~min}$. Ich empfehle eine einmalige Antibiotikagabe (z. B. Cefuroxim $1,5 \mathrm{~g}$ i.v.) $20 \mathrm{~min}$ vor dem Eingriff. Für die rautenförmige Exzision und die Lappenpräparation werden die Gesäßbacken durch Klebestreifen lateralisiert. Diese müssen für das Einschwenken und
Einnähen des Lappens gelöst werden. Als Verband empfehle ich einen kanisterlosen Unterdruck-Wundverband (z.B. PICO-Verband ${ }^{\circledR}$ Smith and Nephew). Leider ist dies bei sehr nahe an den Anus reichenden Lappenplastiken nicht immer möglich. Dann ist ein Verband mit Kompressen komplikationsärmer. Eine subkutan eingebrachte Redondrainage wird für $48 \mathrm{~h}$ belassen. Postoperativ halten die Patienten eine gelockerte Bettruhe (Verlassen des Bettes so wenig wie möglich) für die Hospitalisationsdauer ein. Den Patienten wird empfohlen, bis zum Ende des stationären Aufenthalts möglichst eine Seiten- und Bauchlage einzunehmen, der in der Regel am 4. postoperativen Tag nach Entfernung des Unterdruck- 
Wundverbands erfolgt. Die Fadenentfernung wird auf den 14. postoperativen Tag geplant. Die Wundheilung dauert aus eigener Erfahrung etwa 14 Tage, und die volle Belastbarkeit der Narbe ist nach 4 Wochen erreicht. Je nach beruflicher Tätigkeit haben die Patienten eine 14- bis 21-tägige Arbeitsunfähigkeit. Die asymmetrische Narbe läuft zum Teil quer über das Gesäß und ist bei wenig stark behaarten Patienten gut sichtbar. Diese kann kosmetisch als störend empfunden werden und sollte deshalb vor dem Eingriff erwähnt werden. Gelegentlich berichten Patienten von einem Taubheitsgefühl kaudal der Entnahmestelle des Lappens. Diese Hyposensibilität der Haut erholt sich nach mehreren Monaten spontan. Eine Rezidivprophylaxe durch Epilation der Glutealregion wird nach der Limberg-Plastik nicht empfohlen.

\section{Operationstechnik}

Der Patient ist in Bauchlage. Die beiden Gesäßbacken werden mit Klebestreifen $\left(\mathrm{Mefix}^{\circledR}\right)$ maximal nach lateral gezogen (• Abb. 1).

Einzeichnen der rautenförmigen Exzision. Ober- und Unterpol werden durch die Ausdehnung des Sinus pilonidalis definiert, d.h. alle Pori und Fisteln müssen eingeschlossen sein (• Abb. 2). Der kürzeste Abstand zwischen Ober- und Unterpol beträgt ca. $5 \mathrm{~cm}$, der längste $8 \mathrm{~cm}$. Darunter ist eine Lappenplastik schwierig und darüber kosmetisch heikel. Der Unterpol wird aus der Rima lateral linksseitig geplant. Die Kantenlänge und der quere Durchmesser der Raute entsprechen $60 \%$ des Abstands vom Ober- zum Unterpol. Am Beispiel dieses Patienten: Gemessener axialer Durchmesser $6 \mathrm{~cm}$, davon berechneter querer Durchmesser $3,6 \mathrm{~cm}$, ergibt Kantenlänge $3,6 \mathrm{~cm}$. Entsprechend wird auf der glutealen rechten Seite ein Verschiebelappen eingezeichnet (• Abb. 3).

Die Fisteln können bei Bedarf mit blauem Farbstoff gefüllt werden. Dies ist aus Sicht des Autoren bei Einschluss aller Sinus in die geplante Resektion nicht nötig. Die Exzision muss immer bis auf die Knochenfaszie des Sakrums und Os coccygis reichen. Es erfolgt die sorgfältige

coloproctology 2021 · 43:114-118 https://doi.org/10.1007/s00053-021-00519-3

(c) Springer Medizin Verlag GmbH, ein Teil von Springer Nature 2021

\section{F. H. Hetzer}

\section{Modifizierter Limberg-Lappen beim Sinus pilonidalis}

\section{Zusammenfassung}

Hintergrund. Das Ausmaß der chirurgischen Behandlung des symptomatischen Sinus pilonidalis reicht von minimal-invasiven Verfahren, wie Sinusektomie und Pit-Picking, bis zu maximaler Exzision der Rima ani. Gewebeschonende Verfahren weisen in der Regel eine kürzere Wundheilungszeit, aber leider auch eine höhere Rezidivrate auf. Die radikale Exzision mit offener Wundbehandlung bedarf einer langen Wundpflege. Eine Deckung des Defekts mittels Verschiebelappen kann diese lange Wundheilungsphase nach großer Exzision signifikant verkürzen. Deshalb sind Lappenplastiken, wie z. B. die Limberg- oder Karydakis-Plastik, eine patientenfreundliche Alternative und weisen zudem eine sehr niedrige Rezidivrate auf (0-6\%). Die Plastiken sind aber chirurgisch anspruchsvoll und gemäß Literatur mit einer großen Bandbreite von Wundheilungsstörungen (0-45\%) verbunden.

\begin{abstract}
Methode. Die Exzision erfolgt in Bauchlage unter einmaliger Antibiotikagabe kurz vor dem Eingriff. Die Präparation des Verschiebelappens muss mit maximaler Gewebeschonung vorgenommen werden. Der Lappen beinhaltet auch die Faszie des M. gluteus maximus zur sicheren Refixation. Die Rekonstruktionsnaht sollte mehrreihig und engmaschig sein. Eine postoperative Schonung des Operationsgebiets für 3-4 Tagen ist empfehlenswert.

Schlussfolgerung. Die Limberg-Plastik ist ein anspruchsvolles chirurgisches Verfahren zur Deckung eines radikal exzidierten Sinus pilonidalis. Wegen seiner geringen Morbidität und tiefen Rezidivrate wird das Verfahren u. a. auch in der S3-Leitlinie der AWMF erwähnt und empfohlen.
\end{abstract}

Schlüsselwörter

Pilonidalsinus - Chirurgische Verfahren . Lappenplastik · Limberg-Plastik · Rezidivrate

\section{Modified Limberg flap in pilonidal sinus}

\section{Abstract}

Background. The extent of the surgical treatment of symptomatic pilonidal sinus ranges from minimally invasive procedures, such as sinusectomy and pit picking, to radical excision of the anal fissure. The tissuesparing techniques normally have a shorter wound healing time but the recurrence rate is higher. The radical excision with open wound treatment needs a lengthy wound care. A closure of the defect with a cross-flap can significantly shorten this long healing phase after an extensive excision. Therefore, flap plasty, such as Limberg and Karadakis flaps, are a patient-friendly alternative and show a very low recurrence rate (0-6\%); however, the flap procedures are surgically demanding and according to published studies are associated with a large range of wound healing complications (0-45\%).

Method. The wide excision is performed with the patient in a prone position and a single dose of an antibiotic shortly before the intervention. The preparation of the crossflap has to be done with a very careful tissue handling. The cross-flap includes the fascia of the gluteus muscle for safe refixation. The reconstruction suture should be multirow and close knit. For the first 3-4 days after surgery patients are advised not to strain the gluteal area.

Conclusion. The Limberg flap is a demanding surgical technique for covering the defect after radical excision of a pilonidal sinus. Because of the low morbidity and low recurrence rate it is an excellent and recommended procedure in the $S 3$ guidelines of the Association of the Scientific Medical Societies in Germany (AWMF).

Keywords

Pilonidal disease · Surgical procedures · Flap plasty - Limberg flap - Recurrence rate 


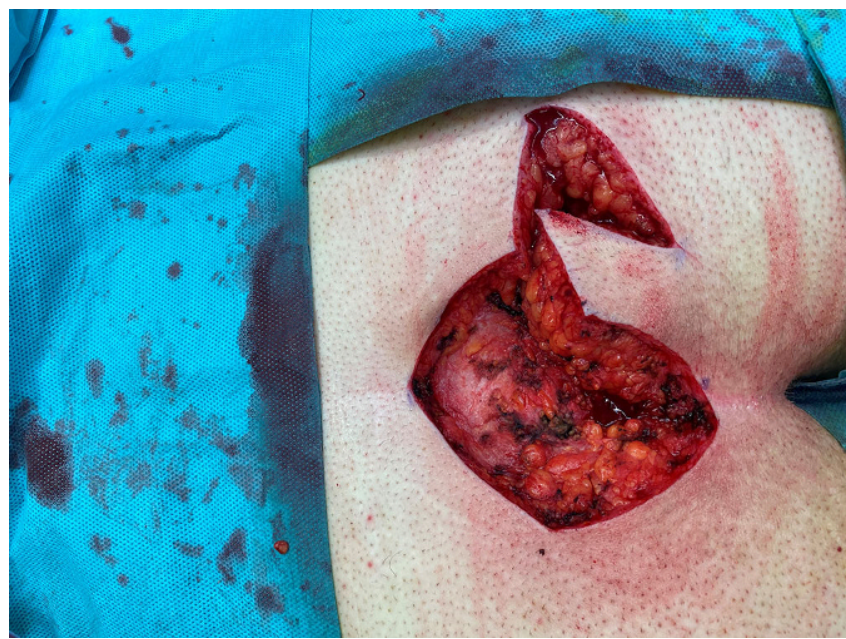

Abb. 5 \ Präparation des Verschiebelappens gluteal rechts

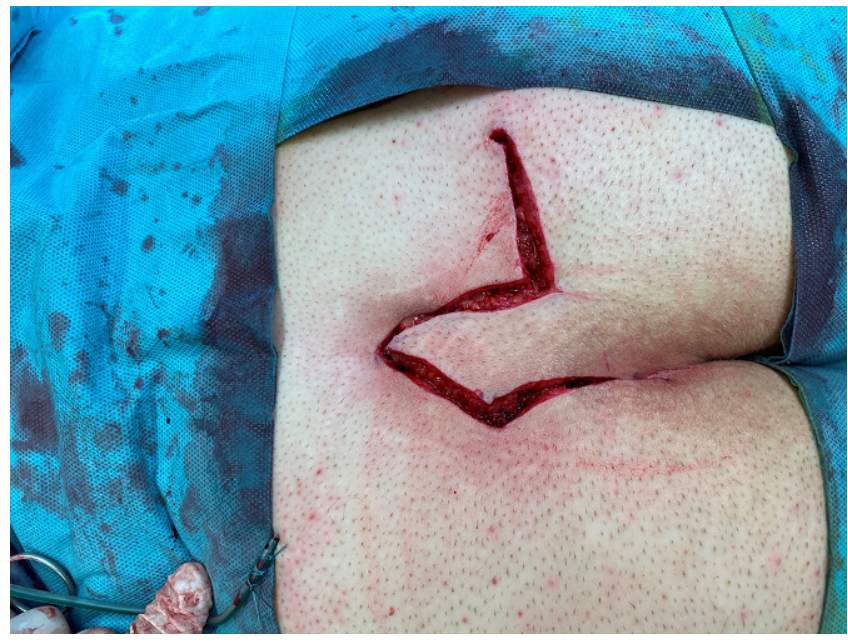

Abb. $7 \Delta$ Nach Einlage einer Redondrainage und Knoten der Fasziennähte

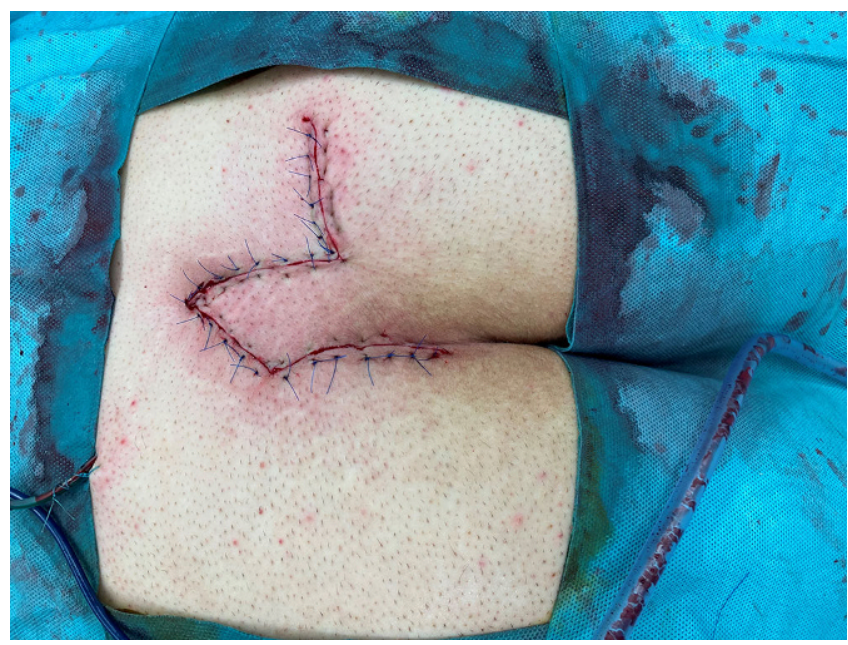

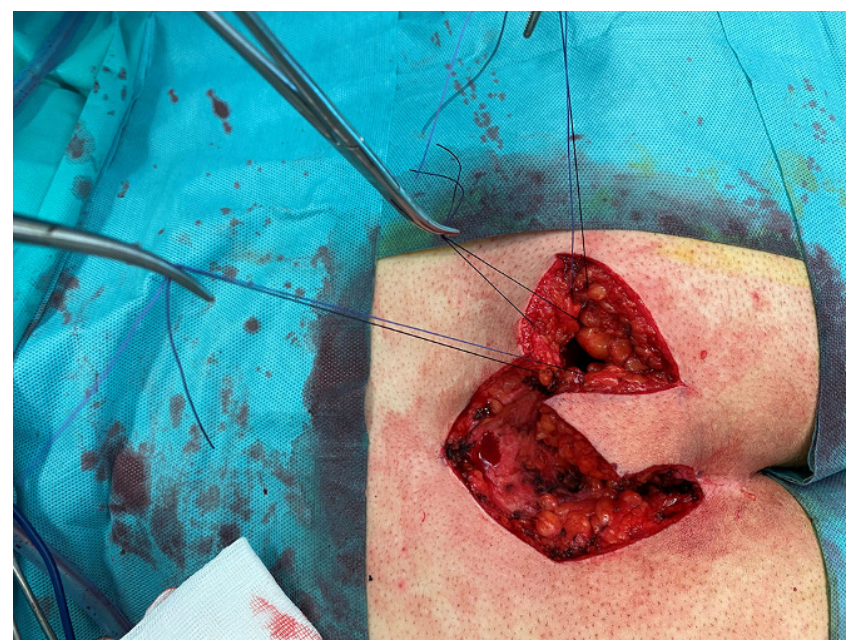

Abb. $6 \Delta$ Transposition des Verschiebelappens in den Defekt und Fixation mit vorgelegten Fasziennähten

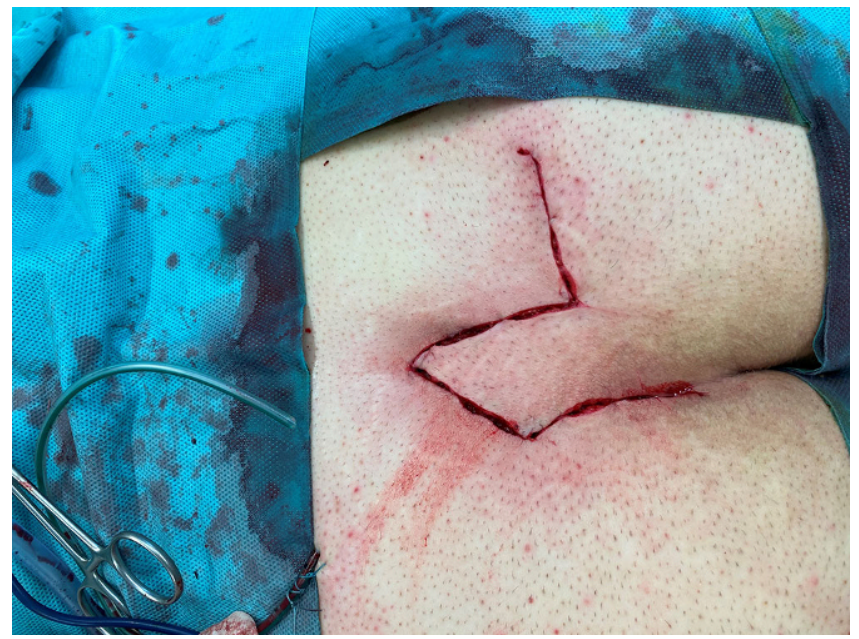

Abb. $8 \Delta$ Situs nach Subkutan- und Intakutannaht

Blutstillung, zudem darf in der Schweiz noch mit $\mathrm{H}_{2} \mathrm{O}_{2}$ gespült werden (• Abb. 4).

\) Der Lappen soll die Faszie des Gluteus maximus beinhalten

Nun wird der Ersatzlappen gluteal präpariert. Die Inzision wird hauptsächlich mit dem Skalpell durchgeführt. Der Einsatz von Koagulation sollte so sparsam wie möglich gehandhabt werden. Hitze oder Quetschen der Spitze des Lappens muss vermieden werden. Es wird empfohlen, die Faszie des Gluteus maximus mitzunehmen, um später ein gutes Nahtlager zu haben (• Abb. 5).

Bevor der Lappen in den Defekt transponiert wird, müssen die Klebebänder
Abb. $9<$ Hautnaht in Donati-Rückstichtechnik 
gluteal beidseits gelöst werden, damit der Lappen spannungsfrei eingenäht werden kann. In einer ersten Nahtreihe werden die Faszien mit Vicryl 2:0 adaptiert. Diese Nähte werden vorgelegt, mindestens zwei pro Kante. Bevor sie geknotet werden, wird eine Redondrainage mit der Spitze auf dem Steißbein eingelegt (• Abb. 6 und 7).

In einer zweiten Nahtreihe wird die Subkutis mit Vicryl ${ }^{\circledR}$ 3:0 adaptiert. Um die abschließende Haut zu entlasten, wird eine dritte intrakutane Nahtreihe mit Vicryl rapid $^{\circledR}$ 4:0 (farblos) vorgenommen (mindestens vier invertierte Einzelknopfnähte pro Kante; • Abb. 8).

Die Hautnaht erfolgt schlussendlich mit Prolene ${ }^{\circledR}$ 4:0 in Donati-Rückstichtechnik. Um eine sichere Wundheilung $\mathrm{zu}$ erreichen, ist sind kleine Abstände zwischen den Nähten (max. $5 \mathrm{~mm}$ ) empfehlenswert (- Abb. 9).

\section{Fazit für die Praxis}

- Die Limberg-Plastik beim Sinus pilonidalis zeichnet sich durch eine rasche Wundheilung und niedrige Rezidivrate aus.

- Die Technik ist einfach, bedarf aber einer sorgfältigen plastisch-chirurgischen Präparation und Rekonstruktion.

- Eine postoperative Schonung mit gelockerter Bettruhe von 3-4 Tagen ist empfehlenswert.

- Die Arbeitsfähigkeit ist in der Regel nach 14 Tagen wieder gegeben.

- Das kosmetische Resultat muss mit den Patienten vor dem Eingriff besprochen werden.

\section{Korrespondenzadresse}

\begin{tabular}{ll} 
& $\begin{array}{l}\text { Prof. Dr. med. } \\
\text { Franc H. Hetzer } \\
\text { Viszeralchirurgie/ } \\
\text { Koloproktologie, Praxis } \\
\text { Bellaria/Klinik im Park } \\
\text { Bellariastraße 38, 8038 Zürich, } \\
\text { Schweiz } \\
\text { viszeralchirurgie- } \\
\text { bellaria@hin.ch }\end{array}$ \\
\hline
\end{tabular}

Einhaltung ethischer Richtlinien

Hier steht eine Anzeige. 望 Springer

Interessenkonflikt. F.H. Hetzer gibt an, dass kein Interessenkonflikt besteht.

Für diesen Beitrag wurden von den Autoren keine Studien an Menschen oder Tieren durchgeführt. Für die aufgeführten Studien gelten die jeweils dort angegebenen ethischen Richtlinien. Für Bildmaterial oder anderweitige Angaben innerhalb des Manuskripts, über die Patienten zu identifizieren sind, liegt von ihnen und/oder ihren gesetzlichen Vertretern eine schriftliche Einwilligung vor.

\section{Literatur}

1. Omer A, Doll D, lesalnieks I (2020) S3-Leitlinie Sinus pilonidalis Kurzfassung, AWMF-Registriernummer:081-009Erstellungsdatum 